\title{
Protective autoimmunity in cancer (Review)
}

\author{
E. TOUBI ${ }^{1}$ and Y.SHOENFELD ${ }^{2}$
}

\begin{abstract}
${ }^{1}$ Division of Allergy and Clinical Immunology, Bnai Zion Medical Center; Affiliated with Technion Faculty of Medicine Haifa; ${ }^{2}$ Department of Internal Medicine 'B' and Center of Autoimmune Diseases, Sheba Medical Center, Tel Hashomer; Affiliated with Sackler Faculty of Medicine; Incumbent of the Laura Schwarz-Kipp Chair for Research of Autoimmunity Diseases, Tel-Aviv University, Israel
\end{abstract}

Received August 31, 2006; Accepted October 2, 2006

\begin{abstract}
Not all malignant cells progress to invasive cancer, some may even regress, but the early detection of abnormal cells can be crucial for patient survival. Immune surveillance mechanisms are complex and provide continuous efforts for the removal of transformed cells. Naturally occurring antibodies are frontier soldiers that act as the first line of defense in the battle against cancer. During the process of carcinogenesis naturally occurring antibody responses to tumor antigens were found to be associated with improved survival and protection against the spread of cancer. Using the human hybridoma technology, a series of tumor-binding antibodies can be isolated as they have several common features: they are germ-line coded $\operatorname{IgM}$ antibodies, they bind to various tumor-antigens, they induce apoptosis of malignant cells, and most importantly they detect not only malignant cells but also the precursor stages (i.e., autoantigens). Natural protective autoantibodies against tumor-antigens were isolated from patients and healthy donors reflecting the development of naturally occurring B-cell responses during the process of cancer evolvement. They fulfill the definition of autoantibodies since they are self-reactive and they also bind altered selfantigens such as tumor cells. In this regard various autoantibodies such as anti-dsDNA and anti-Fas autoantibodies were found to be significantly higher in patients with various carcinomas, thus playing a role for their improved survival. Targeting T-regulatory cells, namely the expression of CTLA-4 was also found to improve survival in cancer patients. Autoimmunity and malignancy frequently coexist and they may share etiological and pathological mechanisms. Therefore, the efficacy of intravenous immunoglobulins or CTLA-4 blockade was also employed as a treatment for prevention of malignancy and metastases spread.
\end{abstract}

Correspondence to: Professor Y. Shoenfeld, Department of Medicine 'B' Sheba Medical Center, Tel-Hashomer 52621, Israel E-mail: shoenfel@post.tau.ac.il

Key words: cancer, autoimmunity, autoantibodies, protection, melanoma, vertigo

\section{Contents}

1. Introduction

2. Immune surveillance mechanisms in cancer

3. Protective humoral responses in cancer

4. CTLA-4 blockade in cancer prevention

\section{Introduction}

For many decades investigators assumed that the immune system (lymphocytes, antibodies and macrophages) can recognize and reject tumor cells by reacting against tumorspecific antigens. However, immunity against these altered self-antigens is weak and unable to efficiently destroy tumor growth (1). A better definition and characterization of these antigens led to the development of potential antigens for cancer vaccines and human cancer immunotherapy. Among the innate anti-tumor immune responses the roles of neutrophils, macrophages and natural killer (NK) cells must be mentioned. Upon activation, neutrophils and macrophages are able to partially kill tumor cells by releasing reactive oxygen substances (ROS) and angiogenic and immune suppressive substances $(2,3)$. However, the adaptive immune system provides the body with a more efficient anti-tumor immune response such as that of cytotoxic T lymphocytes (CTLs), T helper cells, and natural/protective autoantibodies (4).

Historically, immune-based therapies that are canceroriented have focused on eliciting a CTL response, primarily due to the fact that CTL can directly kill tumors. Many putative tumor antigens are intracellular proteins, and CTL respond to peptides presented in the context of MHC class I which are most often derived from intracellular proteins. Recently, greater emphasis has been placed on the stimulation of $\mathrm{CD}^{+} \mathrm{T}$ helper cell (Th) responses in cancer. Th cells are central to the development of an immune response by activating antigen-specific effector cells and recruiting cells of the innate immune system such as macrophages. By secreting cytokines such as IL-2 Th1 cells are primarily responsible for activating and regulating the development of CTL. In addition, the release of other pro-inflammatory cytokines such as TNF- $\alpha$ activates death receptors on the tumor cell surface and kills tumor cells (5-7).

Natural humoral immunity seems to be also important in the detection of transformed cells. The human hybridoma 
technology offered a unique opportunity to study the mechanisms, structures and targets involved in recognition and elimination of aberrant cells. Thousands of tumor-reactive human monoclonal antibodies were isolated by this technique from cancer patients and healthy donors. Most of these antibodies are of the IgM isotypes; they express only a few or no mutations at all (germ-line coded), they are bound to carbohydrates on modified tumor-specific receptors, and they induce tumor cell apoptosis (8). These are naturally occurring autoantibodies (NAA) mostly produced by $\mathrm{CD}^{+} \mathrm{B}$ cells and are extensively reported to play a protective role in preventing the spread of cancer by cross-reacting with both themselves and tumor-antigens (9).

In this review we summarize the current data on protective autoimmunity and its important role in immune surveillance mechanisms against transformed cells in humans.

\section{Immune surveillance mechanisms in cancer}

Naturally occurring T cells directed against tumor-associated antigens (TAAs) can frequently be detected in cancer patients. Immune surveillance was shown to be mediated mainly by tumor-specific CD8 ${ }^{+}$CTLs, however, macrophages, $\mathrm{CD}^{+}$and NK T cells are also involved. These responses were found for various tumor-antigens, including melanoma, colorectal cancer and breast cancer (10). Melanoma cells were recognized and destroyed by autoreactive $\mathrm{T}$ cells (both $\mathrm{CD} 8^{+} \mathrm{CTL}$ 's and $\mathrm{CD}^{+} \mathrm{T}$ cells) since they recognized melanosomal proteins such as the tyrosinaze family (required for the synthesis of melanin in melanocytes). Novel antigen-specific immunotherapeutic strategies in melanoma are currently being studied and point to the importance of enhancing autoreactive $\mathrm{T}$ cell responses directed against this category of altered self-antigens (11).

The requirement for $\mathrm{CD}^{+}$Th cells in the priming of CD8 ${ }^{+}$CTL responses in cancer was investigated (12). It showed that $\mathrm{CD}^{+}$Th cells activated by OVA-pulsed dendritic cells are Th1 phenotype, and that these $\mathrm{CD}^{+} \mathrm{Th}$-APCs stimulate naïve $\mathrm{CD} 8^{+} \mathrm{T}$ cell proliferation through signal I (MHC I/ OVA/TCR) and signal II (CD80/CD28) interactions and IL-2 help. In vivo, they stimulate $\mathrm{CD}^{+} \mathrm{T}$ cell proliferation and differentiation into CTLs and induce effective OVA-specific anti-tumor immunity. This demonstrates that $\mathrm{CD}^{+}{ }^{+} \mathrm{Th}$ cells carrying acquired Ag-presenting machinery can, and alone they efficiently stimulate CTL responses.

Cellular immunity against tumor antigens is suppressed by regulatory $\mathrm{CD} 4{ }^{+} \mathrm{CD} 25^{+} \mathrm{T}$ cells or shift towards a Th2 response. The beneficial balance between a protective immune response against tumor antigens and a suppressive anti-tumor immune response in favor of the former is warranted in order to prevent the development of cancer. A possible mechanism of cancer immune evasion is the suppression of anti-tumor autoimmunity by immune regulatory $\mathrm{T}$ cells i.e., $\mathrm{CD} 4{ }^{+} \mathrm{CD} 25^{+}$ $\mathrm{T}$ cells. They were found to suppress the activation and proliferation of both autoreactive pro-inflammatory $\mathrm{CD}^{+}$and $\mathrm{CD}^{+}$cytotoxic T cells (13). Augmentation of $\mathrm{CD}^{+}{ }^{+} \mathrm{CD} 25^{+} \mathrm{T}$ regulatory cell numbers or proportions in peripheral blood and at tumor sites has been reported in patients suffering from various cancers. The percentage of $\mathrm{CD} 4{ }^{+} \mathrm{CD} 25^{+} \mathrm{T}$ cells in patients with different stages of gastrointestinal malignancies is inversely correlated with disease prognosis. Depletion of $\mathrm{CD}^{+}{ }^{+} \mathrm{CD} 25^{+} \mathrm{T}$ cells in vivo by anti-CD25 antibodies before tumor challenge enhanced natural tumor immune surveillance and induced rejection of many immunogenic tumors in mice (14). In a recent study, the Toll-like receptor (TLR) 8 signaling was linked with the suppressive function of T regulatory cells against cancer cells. It was found that synthetic and natural ligands for human TLR8 could reverse T reg cell function. This effect was independent of dendritic cells but required functional TLR8-MyD88-IRAK4 signaling in T reg cells. Adoptive transfer of TLR8 ligand-stimulated T reg cells into tumor-bearing mice enhanced anti-tumor immunity. These results suggest that TLR8 signaling could play a critical role in controlling immune responses to cancer (15).

The regulatory role of NKT cells has been mentioned primarily in Th1-induced autoimmune diseases. However, NKT cells were also shown to have suppressive anti-tumor immune responses in many mouse tumor models (16). In such models, $\mathrm{CD}^{+} \mathrm{T}$ cells were demonstrated to suppress $\mathrm{CD}^{+}$ CTLs, thus preventing the efficient elimination of tumors. This suppression was shown to be mediated by enhanced IL-13 secretion, which activates myeloid cells to produce TGF- $\beta$ and directly suppress the CTLs function. When IL-13 or TGF- $ß$ was blocked, CTL-mediated tumor killing was enhanced. In humans, different types of cancer were reported to be associated with a reduction of both the NKT-cell population and their shift towards Th2-cytokines (17). Better characterization of the mechanisms that regulate autoreactive anti-tumor immune responses and the ability to manipulate immune regulatory suppressive T and NKT cells may lead to better control of cancer, or to improved efficacy of the vaccine, or to adopting immunotherapy strategies.

\section{Protective humoral responses in cancer}

The adaptive immune system is capable of recognizing cancer through T- and B-cell receptors. However, priming adaptive immunity against self antigens is a potentially difficult task. Immunization with conserved paralogues of tumor antigens can induce adaptive immunity against self antigens expressed by cancer. Cancer immunity and autoimmunity use overlapping mechanisms and are therefore difficult to uncouple, but distinct pathways can be discerned that open the eventual possibility of uncoupling tumor immunity from autoimmunity (18).

Natural autoantibodies in cancer. Natural polyreactive autoantibodies (NAA mainly of IgM isotypes) encoded by unmutated germline $\mathrm{IgV}$ genes represent a major part of the normal circulating antibody repertoire. They fulfill the definition of autoantibodies since they are self-reactive, but they also bind altered self-antigens such as tumor cells. These germ-like coded IgM molecules are produced as mentioned above by $\mathrm{CD}^{+}$lymphocytes and are of pentameric structure, which appears to be important for their functional activity. They do not bind to distinct protein sequences, but recognize specific patterns of conservative molecules. These molecules can be repetitive structures, polysaccharide molecules on bacteria, or carbohydrate residues on tumor-specific cell surface receptors $(9,19,20)$. In some cases both naturally 
Table I. Protective autoantibodies and their targets in cancer.

\begin{tabular}{|c|c|c|c|}
\hline Autoantibody & Target antigen & Function & References \\
\hline $\begin{array}{l}\text { Anti-CENP-B } \\
\text { anti-SS-B }\end{array}$ & Breast cancer & Unknown & $(25)$ \\
\hline $\begin{array}{l}\text { Anti-survivin } \\
\text { anti-livin }\end{array}$ & Breast cancer & Unknown/diagnostic & (26) \\
\hline Anti-thyroglobulin & Thyroid cancer & Unknown & $(28)$ \\
\hline Anti-melanocytes & Melanoma cell lines & Proliferation inhibition & (29) \\
\hline $\begin{array}{l}\text { Antibody mediated Lambert- } \\
\text { Eaton myastenic syndrome }\end{array}$ & Small cell lung cancer & Diagnostic & (30) \\
\hline Natural IgM-autoAbs & Leukemia cells & Apoptosis & (31) \\
\hline Anti-Fas Abs & $\begin{array}{l}\text { Colorectal adeno- } \\
\text { carcinoma cells }\end{array}$ & Apoptosis & $(32)$ \\
\hline Anti-dsDNA & $\begin{array}{l}\text { SP2/0 and Wehi } 164 \\
\text { tumor cells }\end{array}$ & Apoptosis & (34) \\
\hline
\end{tabular}

occurring and vaccine-induced antibody responses to some breast cancer antigens are associated with improved survival. However, the improved survival associated with antibody responses to breast cancer is modest, indicating the need for improved understanding of the natural anti-tumor antibody response. Naturally occurring B-cell responses in the form of serum antibody, tumor reactive lymph node B cells, and tumorinfiltrating B cells have been described and a variety of breast tumor-associated antigens have been identified based on the reactivity of patient antibodies. With few exceptions, the 'tumor-associated antigen' identified with the naturally occurring antibodies may be overexpressed on tumors, but are in fact non-specific autoantigens (21). This suggests that while overexpression or aberrant processing can increase immunogenicity in some cases, most cases were tumorassociated antigens, a function of the tumor expression or the result of ancillary tumor factors. A recent study demonstrated a highly complex but specific humoral immune response against a benign tumor with a distinct serum reactivity pattern and a decline of malignant complexity (22). The frequent antibody response against specific antigens offers new diagnostic and therapeutic targets for meningioma.

In a previous study, five human monoclonal $\operatorname{IgM}$ antibodies from cancer patients were established (23). Antibody LM-1 was isolated from a patient with lung cancer, antibodies PM-1 and PM-2 were isolated from a patient with pancreatic cancer, and antibodies CM-1 and CM-2 were isolated from a patient with colon carcinoma. The mainly germ-line encoded antibodies were specific for malignant tissues and showed only restricted reactivity with healthy cells. When tested for in vitro functional activity, all five antibodies inhibited tumor cell proliferation of carcinoma cells by inducing apoptosis.

Polymorphic epithelial mucin (MUCI) has been studied as a vaccine substrate for the immunotherapy of patients with adenocarcinoma. In this regard, the incidence of naturally occurring MUCI antibodies in early breast cancer patients was analyzed and related to the pretreatment serum of the disease outcome (24). A positive test result for both MUCI $\mathrm{IgG}$ and $\mathrm{IgM}$ antibodies in pretreatment serum was associated with a significant benefit in disease-specific survival in stage I and II ( $\mathrm{p}=0.01)$ breast cancer patients. Positive $\operatorname{IgG}$ and $\operatorname{IgM}$ MUCI antibody levels had significant additional prognostic value to the tumor stage $(\mathrm{p}=0.04)$ in multivariate analysis. Thus early breast cancer patients with a natural humoral response to MUCI have a higher probability of freedom from distant failure and a better disease-specific survival.

Protective autoantibodies in cancer. Sufficient evidence has now been collected to show that innate immunity and natural IgM play a very important role in the primary recognition and elimination of infectious particles and the removal of neoantigens, and also in immune surveillance mechanisms against malignant cells. The understanding of these naturally occurring autoimmune responses against altered-tumor antigens will facilitate the development of strategies for cancer immune prevention (Table I).

Autoantibodies were reported to be present in many malignant diseases. In this respect, anti-SS-B and antiCENP-B autoantibodies were studied in breast cancer patients and compared to a control group surgically treated due to benign diseases. Fifty-five patients with breast cancer and 25 patients with benign diseases were prospectively included in the study. In the breast cancer group, both antiCENP-B (33 vs 8\%) and anti-SS-B (44 vs 24\%) autoantibodies had a higher positivity in comparison to the control group, but this difference reached statistical significance only for anti-CENP-B antibodies $(p=0.02)$. Besides anti-SS-B positivity was detected more frequently in breast cancer patients with axillary involvement (63 vs 24\%) $(\mathrm{p}=0.006)$ and increased as the number of axilla involved 
lymph nodes multiplied ( $\mathrm{p}=0.03$ ). These findings point to the fact that autoimmunity is frequent in malignancy (25).

Survivin and livin are highly expressed in cancer cells and transformed cells, however they show little or no expression in normal differentiated tissues. The prevalence of anti-livin and anti-survivin antibodies in lung cancer patients was determined by using a cut-off value for positivity determined as the mean absorbance $+2 \mathrm{SD}$ for healthy control samples and 19 of 37 lung cancer patients $(51.3 \%)$ were positive for antilivin antibodies. Of 31 samples from the same lung cancer patients, $18(58.1 \%)$ were positive for anti-survivin antibodies. When sera from 31 lung cancer patients were simultaneously assessed, 21 patients (71\%) were positive for survivin, livin, or both. Testing for both antibodies together may prove useful in detecting lung cancer, but more extensive studies are needed to establish the clinical significance of these antibodies (26).

Based on previous observations that patients with small cell lung cancer (SCLC) survive longer if they have the antibody-mediated Lambert-Eaton myasthenic syndrome (LEMS) and the fact that non-tumor LEMS (NT-LEMS) never develop SCLC, a recent study was designed in order to further investigate immunogenetic factors in SCLC-LEMS vs NT-LEMS and study their role in the survival from SCLC (27). Clinical symptoms, antibody titers, HLA types and alleles were studied in 48 British and 29 Dutch Caucasian LEMS patients. Highly significant associations were found in NT-LEMS, which appeared strongest with HLA-B8, and was correlated with a decreased risk of SCLC. Moreover, in SCLC-LEMS patients, HLA-B8 positivity correlated with prolonged survival after LEMS onset. Here, HLA-DR3-B8 or linked alleles can act as a double-edged sword by favoring autoimmune reactions while also enhancing immune surveillance against the SCLC and improve patient survival. Their absence should help clinicians anticipate an underlying SCLC.

In a cross-sectional study there was an increased prevalence of circulating thyroglobulin autoantibodies ( $\mathrm{TgAbs}$ ) in patients with differentiated thyroid carcinoma (DTC). The prevalence of $\mathrm{TgAbs}$ during initial examination was $29 \%$. During follow-up, TgAb levels rose transiently in one-tenth of the patients, but the prevalence of demonstrable $\mathrm{TgAbs}$ decreased to $<10 \%$ after 3 years. The presence of TgAbs in breast cancer cannot in the meantime predict the initial or residual tumor volume and its prognostic role is still unknown (28).

In previous studies, autoantibodies against membranal components of melanocytes were found in the sera of patients with vitiligo by techniques such as immno-fluorescence and immunoblot and shown to be as IgG. Exposure of melanoma cells to these NAA was followed by inhibition of their proliferation capacity (29). In addition, morphologic alterations exemplified by cell detachment from their solid support associated with melanin release were observed in the B16-F10 cells. In mice treated with a purified IgG fraction from sera of patients with vitiligo, metastatic foci in the lungs became significantly less compared to those treated with purified IgG fraction from healthy subjects. This study was the beginning of many later studies that pointed to the presence of natural anti-tumor autoantibodies that have a destructive effect on tumor cells in vitro and in vivo. In a recent study, the prognostic significance of autoimmunity in patients with stage IIB, IIC or III melanoma who were treated with high-dose adjuvant interferon $\alpha-2 b$ was assessed (30). Here the serum was tested for anti-thyroid, anti-nuclear, anti-DNA and anticardiolipin autoantibodies and the patients were examined for vitiligo before and 1, 3, 6, 9 and 12 months following therapy. Using univariate and multivariate regression analyses it appeared that autoimmunity was an independent prognostic marker for improved relapse-free and overall survival $(p<0.001)$. Thus, the appearance of autoantibodies or clinical manifestations of autoimmunity during treatment with interferon was found to be associated with improved survival for patients with melanoma.

Natural IgM pooled from the normal circulating IgM repertoire, have been shown to be protective against lymphoblastoid cell lines by inducing their apoptosis (31). The IgMinduced cell death involved classical features of apoptosis such as nuclear fragmentation and activation of caspases. Treatment of leukemic cells with IgM resulted in the cleavage of poly-(A)DP ribose polymerase, a substrate of caspases, and a reduction in mitochondrial transmembrane potential during the early period of apoptosis induction. Soluble Fas molecules inhibited this NAA induced apoptosis, suggesting the involvement of the Fas pathway. These results allude to a role for normal IgM antibodies in controlling cell death and proliferation and imply a possible therapeutic role for $\operatorname{IgM}$ antibodies in lymphoproliferative disorders.

Previous observations have shown that both apoptosis and the expression of Fas (Apo-1, CD95) are altered during carcinogenesis in the human colon. Thus, it was logical to ask whether serum titers of autoantibodies against Fas showed any modification during adenoma-carcinoma sequence (32). In this regard, patients with colorectal adenoma $(n=38)$, patients with colorectal adenocarcinomas $(n=21)$, and healthy controls $(n=38)$ were studied. Anti-Fas antibody titers were found to be significantly higher in patients with colorectal adenomas than in healthy controls and higher still in patients with colorectal adenocarcinomas. This suggests that anti-Fas autoantibodies play a protective role during carcinogenesis and reflect the existence of natural autoimmune defense mechanisms.

Anti-dsDNA autoantibodies are of both diagnostic and prognostic value in systemic lupus erythematosus. However, these antibodies were also reported to be detected in the sera of patients with various types of cancer as well as with AIDS. Similar to DNA-EBNA, bacterial DNA incorporated with CpG motif and tumor DNA as modified DNA can elicit the production of antibodies to dsDNA. In previous observations it was shown that in cancer patients the presence of antinuclear and anti-dsDNA autoantibodies indicated a better prognosis of cancer suggesting that these autoantibodies may function as potential anti-neoplastic agents (33).

In a recent study, the use of indirect immunofluorescence assay showed that anti-dsDNA autoantibodies could bind to the surface components of tumor cells. In vitro assays showed that immunosera at week 6 from immunized mice displayed significant cytotoxicity to tumor cells compared to that of the negative control. In addition, by flow cytometry and electrophoresis of fragmented DNA, the cytotoxicity may possibly be mediated by apoptosis. The study also demonstrated that the ability of the anti-dsDNA autoantobodies to induce 
A.

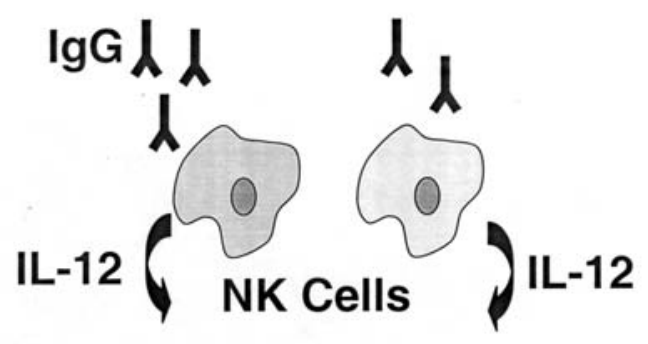

Anti-tumor effect

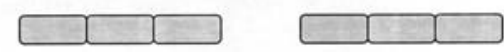

Tumor cells
B.

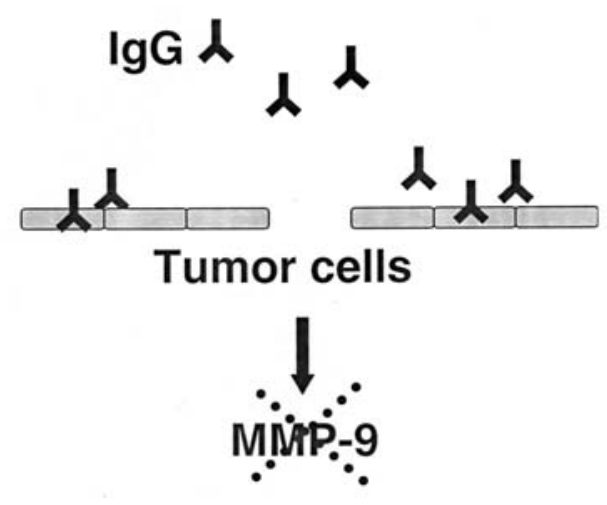

Figure 1. (A), The administration of IVIG was shown to increase natural killer (NK) cell activity and IL-12 production (an anti-tumor and angiogenic effect). (B), MMP-9 is produced mainly by intra-tumor monocytes and is crucial for progression and spread of tumor cells. IVIG was shown to penetrate tumor cells and inhibit MMP-9 production, thus, preventing tumor development.

apoptosis of SP2/0 and Wehi 164 cells was significantly correlated $(\mathrm{r}=0.99, \mathrm{p}<0.01$ and $\mathrm{r}=0.901, \mathrm{p}<0.05)$ with their functional affinity. In vivo, the growth of solid tumors was significantly inhibited in the immunized mice inoculated directly with SP2/0 and Wehi 164 cells, or in the naïve mice inoculated with SP2/0 cells pre-incubated with immunosera containing anti-dsDNA autoantibodies (34). This study points to the origin of anti-dsDNA autoantibodies in cancer patients and tumor-bearing mice, and to the anti-tumor character of these autoantibodies by inducing tumor cell apoptosis. This study supports the notion that ANAs have the potential to act as anti-cancer agents. Apart from being toxic to the tumor cells and their binding capacity to tumor cell membranes and the induction of apoptosis, some studies demonstrated that anti-dsDNA have hydrolyzing activity and can penetrate into living cells.

In another recent study, anti-extractable nuclear antigen (ENA) antibodies were measured in non-Hodgkin's lymphoma patients before, during and after chemotherapy and these values were compared to healthy individuals (35). Data of 66 lymphoma patients were compared with that of 30 healthy controls. The ENA levels were significantly elevated in untreated lymphoma patients compared with the healthy controls ( 1.85 vs $0.68 \mathrm{U} / \mathrm{I}, \mathrm{p}<0.05)$. The patients who responded well to initial chemotherapy demonstrated gradually increasing ENA antibody titers compared to the other patients where a gradual titer decrease was found.

The protective role of IVIG in cancer conditions. High-dose intravenous immunoglobulins (IVIG) is currently used to treat patients with diverse autoimmune conditions. As mentioned above, autoimmunity and malignancy coexist frequently, and may share etiological and pathological mechanisms. Thus, many have studied the efficacy of IVIG for treating malignancy. The administration of IVIG to mice, inoculated with melanoma or sarcoma cells induced a significant inhibition of tumor progression. In this respect, both the number of metastatic lung foci and lung weight were significantly decreased in IVIG treated mice, where neoplastic cells were intravenously inoculated. The administration of IVIG was shown to increase natural killer (NK) cell activity and IL-12 production (of an anti-tumor and anti-angiogenic effect) (Fig. 1A) $(36,37)$. Degeneration of the extracellular matrix (ECM) is crucial for the progression and spread of tumor cells. The ECM-degrading enzymes and matrix metalloproteinases (MMPs) are produced mainly by intra-tumor monocytes. Thus, the reported inhibiting effect of IVIG on MMPs secretion was followed by preventing tumor development. This inhibitory effect on MMP-9 was achieved by F(ab)2 and not by Fc fragments (Fig. 1B). Later, it was reported that $\mathrm{F}(\mathrm{ab}) 2$ prepared from IVIG binds to the cellular structures of different tumor tissues. Positive staining of the cytoplasm and the nuclear membrane of several types of malignant tumors by $F(a b) 2$ from IVIG was also immunohistochemically demonstrated (38). This binding assumes that IVIG treatment of cancer patients may induce an antibody-dependent response against tumors, and suggests that IVIG may become a potentially beneficial adjuvant cancer treatment. This effect was achieved following IVIG or s.c. administration at high dose $(2 \mathrm{~g} / \mathrm{kg}$ body weight) and at 100 times lower doses. In another study (including 200 patients) IVIG was again found to be useful in reducing collagen deposition and preventing metastatic spread (39).

IVIG was able to induce the following chain of events in the amelioration of tumor cells: i) the cross-linking of Fc $\gamma$ receptors on blood effector cells; ii) the release of mediators from these cells, often yielding an infusion-related reaction; iii) the mediator-induced development of a cytokine field characterized by a mutually stabilizing Th2 polarization of CD4 $\mathrm{T}$ lymphocytes and alternative activation of macro- 
anti CTLA- 4 Ab's
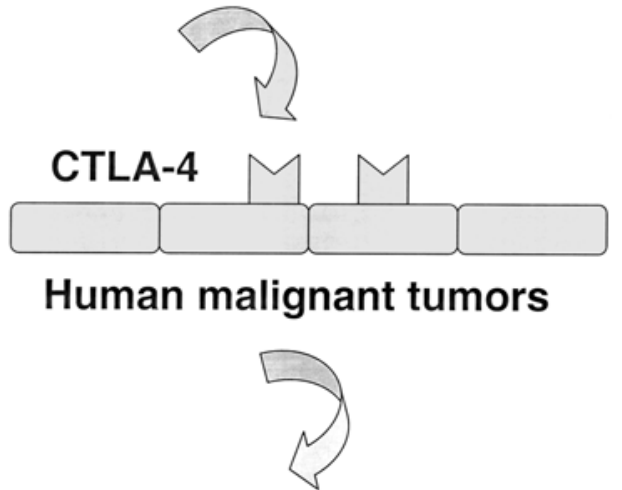

Malignant cell apoptosis

Figure 2. CTLA-4 was found to be expressed on a variety of human malignant cells. Thus, CTLA-4-ligands (antibodies) are effective in inducing malignant cell apoptosis and elimination.

phages; iv) the selective quiescence of these macrophages towards targets coated with $\mathrm{IgG}$ autoantibody due to the increased expression of the macrophage Fc $\gamma$-receptor IIB (40). Thus, it was postulated that in the field of antibody tumor therapy, an undesirable, delayed, or absent subsidence of antibody-coated tumor was due to the immune modulation of the same type as yields amelioration of autoimmunity and arises from a similar chain of events. This chain could usefully be broken at the level of mediator action, indicating that IVIG acts as an anti-tumor agent. Since it has only minor side effects and is used extensively for other clinical conditions, IVIG may be considered as a potential therapy for the prevention of tumor spread in humans.

\section{CTLA-4 blockade in cancer prevention}

Sufficient evidence suggests that tumor growth elicits specific immune responses, including $\mathrm{CD}^{+}$and $\mathrm{CD}^{+} \mathrm{T}$ responses that may delay the tumor growth. As mentioned above, the failure of the immune response, namely the cytolytic action of specific $\mathrm{CD}^{+} \mathrm{T}$ cells that target the tumor may be partially related to the suppressive effect of $\mathrm{CD} 4{ }^{+} \mathrm{CD} 25^{+}$regulatory $\mathrm{T}$ cells. The inhibition of cytotoxicity was found to be dependent on TGF- $\beta$ signaling and CTLA-4 expression by T regulatory cells. Thus, targeting the immune regulation may provide a promising approach to cancer immune therapy. In this regard, autoimmune manifestations and objective cancer regression were reported in patients with metastatic melanoma treated with CTLA-4 blockade (41). Patients with stage IV melanoma or renal cell cancer were treated every 3 weeks with antiCTLA-4 antibodies. Anti-CTLA-4 antibodies did not inhibit the suppressive activity of $\mathrm{T}$ regulatory cells, or decrease the Foxp3 gene expression. However, the percentage of $\mathrm{CD}^{+}$, $\mathrm{CD}^{+} \mathrm{T}$ cells in PBMC expressing the activation marker HLA-DR increased following anti-CTLA-4 antibody administration. This points to the possibility that the antitumor effects of CTLA-4 blockade are due to increased T cell activation rather than the inhibition or depletion of CD $4{ }^{+} \mathrm{CD} 25^{+} \mathrm{T}$ regulatory cells (42). In another study, CTLA-4 was found to be expressed in cell lines derived from a variety of human malignant solid tumors including carcinoma, melanoma and neuroblastoma (43). Treatment of cells from CTLA-4-expressing tumor lines with recombinant forms of the CTLA-4-ligands induced apoptosis associated with caspase 8 and caspase 3 activation. The novel finding that the CTLA-4 molecule is expressed and is functional in human tumor cells opens the possibility of anti-tumor therapeutic intervention based on targeting this molecule (Fig. 2).

The above data support the concept of beneficial autoimmune responses in cancer patients, which could act as a broad spectrum anti-tumor treatment. Future studies should investigate whether the administration of exogenous monoclonal autoantibodies could have a therapeutic effect.

\section{References}

1. Foley EJ: Antigenic properties of methylcholanthrene-induced tumors in mice of the strain of origin. Cancer Res 13: 835-837, 1953.

2. Jakobisiak M, Lasek W and Golab J: Natural mechanisms protecting against cancer. Immunol Lett 90: 103-122, 2003.

3. Hu DE and Brindle KM: Immune cell-induced synthesis of NO and reactive oxygen species in lymphoma cells causes their death by apoptosis. FEBS Lett 579: 2833-2841, 2005.

4. Dhanji S and Teh HS: IL-2-activated CD8 ${ }^{+}$CD44 high cells express both adaptive and innate immune system receptors and demonstrate specificity for syngeneic tumor cells. J Immunol 171: 3442-3450, 2003.

5. Sotiropoulou PA, Perez SA, Voelter V, et al: Natural CD8 ${ }^{+}$T-cell responses against MHC class I epitopes of the HER-2/neu oncoprotein in patients with epithelial tumors. Cancer Immunol Immunother 52: 771-779, 2003.

6. Matsuda K, Tsunoda T, Tanaka H, et al: Enhancement of cytotoxic T-lymphocyte responses in patients with gastrointestinal malignancies following vaccination with CEA peptide-pulsed dendritic cells. Cancer Immunol Immunother 53: 609-616, 2004.

7. Knutson KL and Disis ML: Tumor antigen-specific $\mathrm{T}$ helper cells in cancer immunity and immunotherapy. Cancer Immunol Immunother 54: 721-728, 2005.

8. Vollmers HP and Brandlein S: The 'early birds': natural IgM antibodies and immune surveillance. Histol Histopathol 20: 927-937, 2005.

9. Brandlien S, Pohle T, Ruoff N, Wozniak E, MullerHermelink HK and Vollmers HP: Natural IgM antibodies and immune surveillance mechanisms against epithelial cancer cells in humans. Cancer Res 63: 7995-8005, 2003.

10. Nagorsen D, Scheibenbogen C, Marincola FM, Letsch A and Keilholz U: Natural T cell immunity against cancer. Clin Cancer Res 9: 4296-4303, 2003.

11. Antony PA, Piccirillo CA, Akpinarli A, et al: $\mathrm{CD}^{+} \mathrm{T}$ cell immunity against a tumor/self-antigen is augmented by $\mathrm{CD}^{+} \mathrm{T}$ helper cells and hindered by naturally occurring $\mathrm{T}$ regulatory cells. J Immunol 174: 2591-2601, 2005.

12. Xiang J, Huang $\mathrm{H}$ and Liu $\mathrm{Y}$ : A new dynamic model of $\mathrm{CD}^{+} \mathrm{T}$ effector cell responses via $\mathrm{CD}^{+}{ }^{+} \mathrm{T}$ helper-antigen-presenting cells. J Immunol 174: 7497-7505, 2005.

13. Nomura T and Sakaguchi S: Naturally arising CD4 ${ }^{+} \mathrm{CD} 25^{+}$ regulatory $\mathrm{T}$ cells in tumor immunity. Curr Top Microbiol Immunol 293: 287-302, 2005.

14. Golgher D, Jones E, Powrie F, Elliott T and Gallimore A Depletion of $\mathrm{CD}^{2} 5^{+}$regulatory cells uncovers immune responses to shared murine tumor rejection antigens. Eur J Immunol 32: 3267-3275, 2002.

15. Peng G, Guo Z, Kiniwa Y, et al: Toll-like receptor 8-mediated reversal of $\mathrm{CD}^{+}$regulatory $\mathrm{T}$ cell function. Science 309 : 1380-1384, 2005.

16. Masaki T and Berzofsky JA: Immunoregulatory $\mathrm{T}$ cell in tumor immunity. Curr Opin Immunol 16: 157-162, 2004.

17. Terabe M, Matsui S, Park J-M, et al: TGF-beta production and myeloid cells are an effector mechanism through which CD1drestricted T cells block CTL-mediated tumor immunosurveillance: abrogation prevents tumor recurrence. J Exp Med 198: 1741-1752, 2003 . 
18. Guevara-Patino JA, Turk MJ, Wolchok JD and Houghton AN: Immunity to cancer through immune recognition of altered self: studies with melanoma. Adv Cancer Res 90: 157-177, 2003.

19. Houghton AN, Uchi $\mathrm{H}$ and Wolchok JD: The role of immune system in early epithelial carcinogenesis: B-ware the doubleedged sword. Cancer Cell 7: 403-405, 2005.

20. Vollmers HP and Brandlein S: Death by stress: natural IgMinduced apoptosis. Methods Find Exp Clin Pharmcol 27: 185-191, 2005.

21. Coronella-Wood JA and Hersh EM: Naturally occurring B-cell responses to breast cancer. Cancer Immunol Immunother 52: 715-738, 2003.

22. Comtesse N, Zippel A, Walle S, et al: Complex humoral immune response against a benign tumor: frequent antibody response against specific antigens as diagnostic target. Proc Natl Acad Sci USA 102: 9601-9606, 2005.

23. Brandlein S, Lorenz J, Ruoff N, et al: Human monoclonal IgM antibodies with apoptotic activity isolated from cancer patients. Hum Antibodies 11: 107-119, 2002.

24. Von Mendorff-Pouilly S, Verstraeten AA, Kenemans P, et al: Survival in early breast cancer patients is favorably influenced by a natural humoral immune response to polymorphic epithelial mucin. J Clin Oncol 18: 574-583, 2000.

25. Atalay C, Atalay G, Yilmaz KB and Altinok M: The role of anti-CENP-B and anti-SS-B antibodies in breast cancer. Neoplasma 52: 32-35, 2005.

26. Yagihashi A, Asanuma K, Kobayashi D, et al: Detection of autoantibodies to surviving and livin in sera from lung cancer patients. Lung Cancer 48: 217-221, 2005.

27. Wirtz PW, Willcox N, van der Silk AR, et al: HLA and smoking in prediction and prognosis of small cell lung cancer in autoimmune Lambert-Eaton myasthenic syndrome. J Neuroimmunol 159: 230-237, 2005.

28. Gorges R, Maniecki M, Jentzen W, et al: Development and clinical impact of thyroglobulin antibodies in patients with differentiated thyroid carcinoma during the first 3 years after thyroidectomy. Eur J Endocrinol 153: 49-55, 2005.

29. Fishman P, Azizi E, Shoenfeld Y, et al: Vitilligo autoantibodies are effective against melanoma. Cancer 72: 2365-2369, 1993

30. Gogas H, Ioannovich J, Dafni U, et al: Prognostic significance of autoimmunity during treatment of melanoma with interferon. N Eng J Med 354: 709-718, 2006.
31. Varambally S, Bar-Dayan Y, Bayry J, et al: Natural human polyreactive IgM induce apoptosis of lymphoid cell lines and human peripheral blood mononuclear cells. Intern Immunol 16: 517-524, 2004.

32. Reipert BM, Tanneberger S, Pannetta A, et al: Increase in autoantibodies against Fas (CD95) during carcinogenesis in the human colon: a hope for the immunoprevention of cancer. Cancer Immunol Immunother 54: 1038-1042, 2005.

33. Torchilin VP, Iakoubov LZ and Estov Z: Antinuclear autoantibodies as potential antineoplastic agents. Trends Immunol 22: 424-427, 2001.

34. Lv S, Zhang J, Wu J, Zheng X, Chu Y and Xiong S: Origin and anti-tumor effects of anti-dsDNA autoantibodies in cancer patients and tumor-bearing mice. Immunol Lett 99: 217-227, 2005.

35. Gergely L, Danko A, Csipo I, et al: Antibodies against extractable nuclear antigen in non-Hodgkin lymphoma patients. Scand J Immunol 61: 343-346, 2005.

36. Shoenfeld Y and Fishman P: Gamma-globilin inhibits tumor spread in mice. Int Immunol 11: 1247-1252, 1999.

37. Krause I and Shoenfeld Y: Intravenous immunoglobulin treatment for fibrosis, atherosclerosis, and malignant conditions. Methods Mol Med 109: 403-408, 2005.

38. Fishman P, Bar-Yehuda S and Shoenfeld Y: IVIG to prevent tumor metastases. Int J Oncol 21: 875-880, 2002.

39. Shoenfeld Y and Krause I: IVIG for autoimmune, fibrosis, and malignant conditions: our experience with 200 patients. J Clin Immunol 24: 107-114, 2004.

40. Stevenson GT: Is antibody therapy of tumor compromised by infusion-related reactions? A case for inhibiting the activity of cyclooxygenase-2. Leuk Res 29: 239-246, 2005.

41. Marker AV, Phan GQ, Attia P, et al: Tumor regression and autoimmunity in patients treated with cytotoxic T lymphocyteassociated antigen 4 blockade and interleukin 2: a phase I/II study. Ann Surg Oncol 12: 1005-1016, 2005.

42. Marker AV, Attia P and Rosenberg SA: Analysis of the cellular mechanism of antitumor responses and autoimmunity in patients treated with CTLA-4 blockade. J Immunol 175: 7746-7754, 2005.

43. Contardi E, Palmisano GL, Tazzari PL, et al: CTLA-4 is constitutively expressed on tumor cells and can trigger apoptosis upon ligand interaction. Int J Cancer 117: 538-550, 2005. 